- $\quad<27$ weeks gestational age and/or birthweight $<1000$ grams

- Born between 01/01/2013 - 30/06/2013 and 01/01/2018-30/ 06/2018

- Required mechanical ventilation during the same period.

- The use of the extubation checklist in 2018 group

Results Amongst the pre-checklist cohort, 24 babies met the GA/BW criteria, with 3 sets of notes unavailable. Of these, 15 patients received mechanical ventilation. There were 27 extubation attempts, 19 of these were unsuccessful and 8 of them were successful (29.6\%).

In 2018, 31 babies met the GA/BW criteria, with 4 sets of notes unavailable, 17 patients needed ventilation, 30 extubation attempts using the extubation checklist, 16 of these were unsuccessful and 14 of them were successful (46.6\%)

Conclusions

- It was noted that the proportion of unsuccessful extubation attempts dropped by $17 \%$ (from $70.4 \%$ to $53.4 \%$ ) after implementing the extubation checklist.

- Extubation of extreme preterms is 1.6 more likely to be successful when extubation checklist is used.

\section{DO NEURAMINIDASE INHIBITORS IMPROVE OUTCOMES IN CRITICALLY UNWELL CHILDREN WITH INFLUENZA?}

Amedine Duret, Amedine Duret, Nikita Punjabi. UK

\subsection{6/bmjpo-2021-RCPCH.77}

Background Influenza is a common seasonal acute respiratory viral illness. Children, especially those with co-morbidities, are at risk of complications and ICU admission. No specific guidelines have been formulated about starting neuraminidase inhibitors (NAI) in critically ill children with influenza, but the Health Protection Agency and American Academy of Pediatrics have both stated that antiviral therapy should be initiated as soon as possible in this cohort.

Objectives We aimed to evaluate the evidence supporting the early initiation of NAI in critically ill children with influenza, by conducting a literature search to establish whether NAI improved survival and shortened intensive care admissions in children critically ill with influenza.

Methods We searched the literature for articles on the use of NAI treatment in critically unwell children or children in PICU diagnosed with influenza. We excluded articles with adult patients only, or with a mixture of adults and children where the results were not stratified by age. We also excluded articles with children in outpatient settings, or hospitalised on low-dependency units.

Results Out of 369 articles (Cochrane Library: 2, PUBMED: 328, NHS Evidence: 39), twelve studies published between 2010 and 2017 were included, seven of which were cohort studies (Level 3 evidence) and five case series (Level 4 evidence), with a total of over 7,000 critically ill children with influenza worldwide.

Six cohort studies compared mortality in children receiving NAI and children who did not: five of these demonstrated a trend towards decreased mortality with the use of NAI, and one showed no difference between treated and untreated groups. Only one study reached statistical significance, with $\mathrm{p}$ $=0.01$ for association of NAI treatment with survival. We noted that the two studies which reported on NAI-related adverse events reported none.
There is some evidence in our data that early NAI within 48 hours of symptom onset and/or admission has additional benefits compared to late NAI, although the largest cohort study did not demonstrate this effect.

The studies included here had limitations. Patient cohorts were heterogeneous, with some having had RT-PCR confirmed influenza and others just a clinical diagnosis. Few studies reported on concurrent treatment with antibiotics or steroids. Several studies stressed that patients receiving NAI were more likely to have co-morbidities and very severe influenza requiring mechanical ventilation at baseline, compared to patients who were not started on NAI.

Conclusions The current evidence on the use of NAI in critically ill children with influenza is weak (Level 3), but trends toward improved survival. The trend of improved survival is particularly salient if NAI treatment is initiated within 48 hours of symptom onset. This may mean starting oseltamivir on clinical suspicion of influenza, without waiting for a laboratory confirmation of the diagnosis, to avoid delay.

\section{EARLY POSTNATAL MATERNAL DEPRESSIVE SYMPTOMS MAY PREDICT BEHAVIOURAL PROBLEMS AND AUTISM SYMPTOMS IN TODDLERS}

Ira Kleine, George Vamvakas, Alexandra Lautarescu, Andrew Pickles, David Edwards, Chiara Nosarti. UK

\subsection{6/bmjpo-2021-RCPCH.78}

Background Maternal depression has been studied as a risk factor for child development and found to be associated with less secure attachment, worse cognitive and behavioural outcomes in childhood, and a possible increased risk of autism spectrum disorder (ASD). Despite the wealth of research investigating postnatal parental depression, most studies have small sample sizes, assess parental mood during later infancy, use a dichotomous measure of parental depression, or examine distant child behavioural and emotional outcomes, with limited focus on ASD.

Objectives This study examined the association between early maternal postnatal depressive symptoms and offspring's mental health in a large cohort of term and preterm toddlers.

Methods Participants were 509 children enrolled in the Developing Human Connectome Project; 412 (80.9\%) were born at term, and $97(19.1 \%)$ were born preterm (<37 weeks gestation). Maternal postnatal depressive symptoms were assessed with the Edinburgh Postnatal Depression Scale (EPDS) at term. Children were followed-up at a median corrected age of 18.4 months (range 17.3 - 24.3) for neurodevelopmental assessment. Primary outcome measures were toddlers' Child Behaviour Checklist $1^{1 / 2}-5$ Total (CBCL) and Quantitative Checklist for Autism in Toddlers (Q-CHAT) scores. Secondary outcome measures were the CBCL internalising and externalising sub-scores. Cognition was assessed with the Bayley Scales of Infant and Toddler Development - Third Edition (BayleyIII). Multiple imputation $(n=40)$ was carried out to account for missing data. Data were analysed with multiple linear regression, including clinical and socio-demographic confounders.

Results Higher maternal EPDS scores were associated with toddlers' higher CBCL total scores $(B=0.93$, 95\% CI 0.43 $\left.1.44, \mathrm{p}<0.001, \mathrm{f}^{2}=0.05\right)$ and higher $\mathrm{Q}$-CHAT scores $(\mathrm{B}=0.27$, $95 \%$ CI $\left.0.03-0.52, \mathrm{p}<0.05, \mathrm{f}^{2}=0.01\right)$. Higher maternal EPDS 
score was associated with both internalising $(B=0.22,95 \% \mathrm{CI}$ $\left.0.08-0.36, \quad \mathrm{p}<0.01, \mathrm{f}^{2}=0.03\right)$ and externalising symptoms $\left(\mathrm{B}=0.40,95 \%\right.$ CI $\left.0.20-0.61, \mathrm{p}<0.001, \mathrm{f}^{2}=0.05\right)$, but not with cognitive outcomes in toddlers $(B=-0.21$, 95\% CI $-0.49-$ $0.06, \mathrm{p}>0.05)$. Maternal EPDS, and toddlers' CBCL and QCHAT scores did not differ between preterm and term participants.

Conclusions Our findings indicate that children whose mothers had increased depressive symptoms in the early postnatal period exhibit more maternally-reported behavioural, emotional and neurodevelopmental problems in toddlerhood. Further research is needed to confirm the clinical significance of these findings.

\section{CONTINUOUS INFUSION OF VANCOMYCIN IMPROVED THERAPEUTIC LEVELS IN TERM AND PRETERM INFANTS}

Antonia Round, Khuen Foong Ng. UK

\subsection{6/bmjpo-2021-RCPCH.79}

Background Growing evidence suggests that continuous infusion of vancomycin (CIV) is superior to intermittent infusion of vancomycin (IIV) in neonates. Prescribing CIV in neonates in the UK remains relatively untested and is an unlicensed use. This quality improvement (QI) project aimed to transition from IIV to CIV with earlier and improved attainment of therapeutic vancomycin levels.

Objectives This project aimed to clarify the utility, benefit and safety of CIV over current IIV usage in our department, aiming to inform future vancomycin regimens both locally and more widely. Following baseline measurements of IIV, a QI project was undertaken for implementation of CIV using sequential Plan Do Study Act (PDSA) cycles over 10 months (July 2019 to May 2020) with Specific, Measurable, Achievable, Realistic and Timely (SMART) aims to:

1. Reduce the time taken to achieve vancomycin target level by $50 \%$.

2. To increase first TDM levels within target range from $36 \%$ to $60 \%$.

3. To increase total TDM therapeutic levels from $44 \%$ to $70 \%$ in neonates.

If successful, this would add to current evidence that CIV is superior to IIV in neonates and should be regarded as best practice.

Methods The Model for Improvement framework with Plan Do Study Act cycles was used. Prospective data were collected during three phases: IIV, CIV-1 and CIV-2.

Results Using IIV, 36\% (9/25) of first vancomycin levels were within target range. CIV achieved therapeutic levels twice as quickly as IIV $(\mathrm{p}<0.05)$ with improved first vancomycin target levels (IIV 36\%, 9/25; CIV-1 55\%, 16/29; CIV-2 61\%, $14 / 23$ ) and total therapeutic levels (IIV 44\%, 37/84; CIV-1 56\%, 55/98; CIV-2 69\%, 79/114).

Conclusions It was established that IIV outcomes were not meeting expected standards, particularly in terms of delayed time to therapeutic levels and high percentage of out of range levels. This project was successful in establishing a change to CIV which produced a modest benefit over IIV, particularly in regard to time taken to achieve vancomycin target levels and proportion of therapeutic levels. The findings of this project and information regarding our implementation process provide guidance to other NICUs to transition from IIV to CIV.

\section{HOSPITAL MORTALITY IN PATIENTS WITH RARE DISEASES DURING THE COVID-19 AND SARS PANDEMICS: RESULTS FROM A 7.5 MILLION POPULATION}

Claudia Ching Yan Chung, Wilfred Hing Sang Wong, Brian Hon Yin Chung. Hong Kong

\subsection{6/bmipo-2021-RCPCH.80}

Background The threat of pandemics occur differently for different groups. The rare disease population is at particular risk of being further marginalised during pandemics.

Objectives To assess hospital mortality in the rare disease and general populations during the coronavirus disease of 2019 (COVID-19) and severe acute respiratory syndrome (SARS) pandemics in the 7.5 million population in Hong Kong.

Methods Using the Clinical Data Analysis and Reporting System (CDARS), a population-level database that records all public healthcare records in Hong Kong, all admission records during the COVID-19 (January 23 - August 23, 2020) and SARS (March 11 - June 30, 2003) pandemics were extracted. Patients with rare diseases were identified using one or more of the 1,084 10th version International Classification of Diseases and Related Health Problems (ICD10) codes cross-referenced with 467 ORPHAcodes. Admission records during the same period in 2019 and 2002 were retrieved for comparison. Primary outcomes were COVID-19/ SARS mortality in hospital. Secondary outcomes were overall hospital mortality during the COVID-19 and SARS pandemic periods. Subgroup analysis by age-group $(\leq 18,>18$ to $<60$, $\geq 60$ ) was performed to understand the mortality patterns. Logistic regression was used to estimate the odds ratios with 95\% confidence intervals (CIs).

Results During the COVID-19 pandemic, 407,219 patients were admitted to one or more of the 43 public hospitals in Hong Kong, of which, 39,576 (9.7\%) were paediatric patients $\leq 18$ years old, and $13,894(3.4 \%)$ were rare disease patients. Of the 4,381 patients admitted with COVID-19, $81(1.8 \%)$ died during the same admission, of which, 5 (6.2\%) were patients with rare diseases. COVID-19-related mortality was almost exclusively seen in patients $\geq 60$ years in both rare disease and general populations, with mortality being $21.7 \%$ and $7.4 \%$, respectively. None of the COVID19 patients $\leq 18$ years died by the time of data extraction. Patients with rare diseases had an adjusted 3.4 times odds of COVID-19-related hospital mortality compared with that of the general population (95\% CI 1.24-9.41). In contrast, 158,930 patients were admitted during the SARS pandemic, with $24,045(15.1 \%)$ being $\leq 18$ years and 5,249 (3.3\%) being rare disease patients. Of the 1,449 patients admitted with SARS, $234(16.1 \%)$ died during the same admission, of which, $1(0.4 \%)$ was $\leq 18$ years, and $6(2.6 \%)$ were patients with rare diseases. While age-related increase in mortality was observed for the general population during the SARS pandemic, this pattern was not observed in the rare disease population. Rare disease patients $\leq 18$ years had a 12.5 times higher SARS-related mortality than those in the general population $(12.5 \%$ vs $1.0 \%)$. Patients admitted during the same pandemic periods without coronavirus infection had a significantly higher hospital mortality 